\title{
THE EFFECT OF (COD LIVER OIL-INSULIN) COMBINATION ON SUPPRESSING THE DIABETES DELETERIOUS EFFECT ON THE EXCRETORY DUCTS OF SUBLINGUAL SALIVARY GLANDS OF ALBINO RATS
}

\author{
Marwa M. Abd Elhameed*
}

\begin{abstract}
Background: Diabetes is usually associated with impaired function of salivary glands, by both histolological deterioration and altered salivary composition. Abnormal apoptosis has been recognized in salivary glands of diabetic rats. Amyloidosis constitutes a group of diseases in which proteins deposit in tissues as insoluble fibrils, causing progressive organ dysfunction. Insulin and oral hypoglycemic agents are the widely used in diabetes treatment, but they have undesirable sequelae and fail to completely alter the course of diabetic complications. Cod liver oil (CLO) as an important source of omega-3 ( $\omega-3)$ fatty acid, has been documented to have a powerful antioxidant effect on several tissues.
\end{abstract}

Objective: The present study was carried out to study the possible effect of CLO combined with insulin in regeneration of the exceretory duct of sublingual salivary glands in streptozotocin (STZ)-induced diabetic rats.

Design: forty adult male albino rats (200-250 gm) were selected for this study. The animals were randomly divided into four groups (ten rats each): Group I (Control group), Group II (Diabetic untreated group), Group III (Insulin treated group) and Group IV (Cod liver oil - insulin treated group). At the end of the experimental period (four weeks), the rats were killed and the sublingual salivary glands were dissected out. The sections were examined histologically, immunohistochemically, histomorphometrically and by fluorescence staining technique.

Statistical analysis: Data obtained from histomorphometric analysis were statistically described in terms of mean \pm standard deviation $( \pm \mathrm{SD})$.

Results: Histopathologic examination of Group I showed the normal histological features of the gland's duct system. Group II revealed disturbed ductal outlines, ill-defined ductal cells boundaries, nuclear changes, ductal cells degeneration and stagnated salivary secretion in the lumina. Moreover apparent decrease, hyalinization and degeneration in the surrounding connective tissue (C.T) stroma; fibroblasts showed signs of degeneration and dilated blood vessels (BVs) with swollen endothelial cells. Group III showed better histological features than those

\footnotetext{
* Lecturer, Oral Biology Department, Faculty of Dentistry, Ain Shams University.
} 
of Group II, while Group IV showed histological features nearly resembling those of the control group (Group I). The least immuno-expression for caspase-3 (apoptosis marker) and the minimum fluorescence with thioflavin-T (amyloidosis marker) stain were demonstrated in Group I, followed by Group IV, then Group III and subsequently Group II. The histomorphometric analysis supported the previous results. On the other hand, Group I showed the least mean area percentage of both caspase-3 immunoreactivity and thioflavin-T fluorescence, followed by Group IV, then Group III and the highest values were for Group II. There was statistically highly significant difference between the studied groups.

Conclusions: Diabetes has deleterious effect on the structure of the exceretory duct of sublingual salivary glands. Moreover, it has a major role in tissue damage through development of amyloidosis. Insulin as a sole treatment, couldn't completely inhibit the complications of diabetes. However, CLO- insulin combination had great potential to minimize the abnormalities caused by diabetes.

KEY WORDS: Diabetes, salivary gland, apoptosis, amyloidosis, insulin, cod liver oil.

\section{INTRODUCTION}

Diabetes mellitus (DM) is a metabolic disorder accompanied by impairment of carbohydrate, fat and protein metabolism. This is caused by either decreased sensitivity of the tissues to insulin or lack of insulin secretion. The prevalence of diabetes is uprising rapidly and the disease incidence in 2010 was about 285 million people worldwide and is expected to be increased to 438 million in 2030 . There are two forms of DM: type I and II. In type I diabetes or insulin-dependent DM, the pancreatic $\beta$-cells are progressively destroyed and secrete little or no insulin. Type II diabetes or non-insulindependent DM, is a heterogeneous disorder of insulin resistance and pancreatic $\beta$-cell dysfunction (American Diabetes Association, 2009; Chapple and Genco, 2013).

The diabetic condition is usually accompanied by many disorders as nephropathy, retinopathy, neuropathy, peripheral vascular disease and cardiovascular disease (Kidambi and Patel, 2008). Periodontal disease, gingivitis, tongue inflammation, oral candidiasis, dental caries and xerostomia also affect patients with diabetes. These disorders are generally accompanied with impaired functions of salivary glands, investigated by both morphological deterioration of the glandular parenchyma and by altered salivary composition (Carda et al., 2006; Negrato and Tarzia, 2010). Several reports had documented an altered concentration of proteins such as amylase, lactoferrin and proline-rich protein in diabetic saliva (Aydin, 2007). Furthermore, salivary proteins synthesis is influenced by hyperglycemia (Hirtz et al., 2006; Wong, 2006; Rao et al., 2009; Gregersen and Bross, 2010).

The term apoptosis is given to a programmed cell death. In terms of tissue kinetics, apoptosis could be considered a mechanism that counter balances the effect of cell proliferation by mitotic division. On the other hand, excessive apoptosis might cause organ atrophy and failure. Apoptosis has a major role in several diabetic complications. These include apoptosis of neuronal cells in diabetic neuropathy, myocardial apoptosis which plays a role in cardiac pathogenesis and apoptosis of mesangial cells that occurs in diabetic nephropathy. Furthermore, abnormal apoptosis has been reported in salivary glands of diabetic rat models. The molecular events regulating apoptosis are complex and involve genes that are both pro-apoptotic and anti-apoptotic. Caspases, a family of cysteine proteases, are integral parts of the apoptotic pathway. Fourteen caspases have been identified in the apoptotic pathway cascade. Among these, caspase- 3 is considered to be a major execution protease. Caspase-3, in particular, 
has many cellular targets and when it is activated, it produces morphologic features of apoptosis (Take et al., 2007; Shredah and El-Sakhawy, 2014).

Amyloidosis constitutes a group of diseases in which proteins deposit in tissues as insoluble fibrils, leading to gradual organ dysfunction. About, proteins that are known to cause amyloidosis are unrelated and called amyloidogenic "precursor proteins". The initial step in amyloid fibril formation is a misfolding event. The misfolding can result from proteolytic cleavage, an amino acid substitution or intrinsic properties that become significant only at high serum concentration or in the presence of specific local factors (Merlini and Bellotti, 2003; Westermark et al., 2005). Classification of the amyloidosis is based on the precursor protein that forms the amyloid fibrils and the distribution of amyloid deposition as either systemic or localized. The major types of systemic amyloidosis are immunoglobulin (Ig) light chain (AL), Ig heavy chain (AH), amyloid A (AA), the familial or hereditary amyloidosis (TTR, fibrinogen A $\alpha$, lysozyme, apolipoprotein AI [apoAI], apoAII, gelsolin and cystatin), senile systemic amyloidosis and $\beta 2 \mathrm{~m}$ amyloidosis (Skinner et al., 2004; Obici et al., 2005). It was reported that there was a related events between diabetes type II and the development of amyloidosis (Border et al., 2012; Iannuzzi et al., 2015). Amyloid fibrils could be detected by some fluorescent dyes such as thioflavin- $\mathrm{T}$, producing a characteristic apple green birefringence when visualized under fluorescence microscope (Fabian et al., 2008).

Although insulin and oral hypoglycemic agents are the mainstays of diabetes treatment, they have prominent side effects. Hypoglycemia, macrovascular complications and retinal functions in addition to neuropathic problems are still associated in the patients receiving insulin. Moreover, it has been shown that insulin is unable to completely inhibit protein glycation which can maintain increased oxidative stress in diabetic tissues. These problems led several investigators to focus their attention on the traditional medicines (Jain et al., 2006).

Food derived antioxidants have a strong potential for long term use as chemo-preventive agents in disease states involving oxidative stress (Mckim et al., 2002). There is low prevalence of diabetes in people living in Faeroe Islands, Alaskan Eskimos and the Greenland (Simonsen et al., 1987; Burrows et al., 2000; Jorgensen et al., 2002). The lower prevalence of diabetes in these populations was due to their higher intake of omega-3 ( $\omega-3)$ or $(\mathrm{n}-3)$ rich fish and marine mammals (Jorgensen et $a l ., 2002)$. Fish oil (FO) is a compound rich in $\omega-3$ fatty acids which are polyunsaturated fatty acids (PUFAs) mainly represented by eicosapentaenoic acid (EPA) and docosahexaenoic acid (DHA) that regulate a wide range of functions in the body including blood pressure, blood clotting, modulation of inflammatory response as well as correct development and functioning of brain and nervous systems. Epidemiological studies suggest that among populations ingesting large amounts of PUFAs, mainly present in FO, there are reduced risk of neurodegenerative disorders such as Alzheimer's disease, lower incidence of acute myocardial infarction and chronic inflammatory diseases such as rheumatoid arthritis, ulcerative colitis and psoriasis among other inflammatory diseases (Lordan et al., 2011). Additionally, it has been tested that an increased intake of $\omega-3$ fatty acids might reduce the risk of developing diabetes in mice received streptozotocin (STZ), (Soltan, 2012).

Among the different types of FO, cod liver oil (CLO) is an important source of long-chain $\omega-3$ fatty acids (EPA \& DHA) as well as vitamins A, E and D. Oral administration of CLO improved the deleterious effects of alloxan-induced diabetic rats (Hamdy et al., 2007). Moreover, it was reported that CLO has antioxidant effect especially on parotid glands where an increase of glutathione and catalase 
activities was reported. Furthermore, an increase of catalase activity in submandibular salivary glands was noticed after CLO administration (Leite et al., 2014).

The present study was aimed to investigate the possible role of CLO combined with insulin in enhancement of excretory ducts of sublingual salivary glands in STZ-induced diabetic rats.

\section{MATERIALS AND METHODS}

\section{Animals}

Forty adult male albino rats weighing (200$250 \mathrm{gm})$ were selected for this study. The steps of experiment were done according to the rules approved by the Bio-ethical Committee of Faculty of Dentistry, Ain-Shams University. The rats were housed in separate metal cages, five rats per cage in Ain-Shams animal house under controlled temperature, humidity and dark-light cycle. The size of the cage was $20 \mathrm{~cm}$ width and $40 \mathrm{~cm}$ length. This was done under supervision of specialized veterinarian since their housing till getting rid of sacrificed bodies which was done by the incinerator of Ain-Shams. Rats were kept under good ventilation and adequate stable diet consisting of fresh vegetables, dried bread and tap water throughout the experimental period.

\section{Experimental procedure}

After one week acclimatization period, the animals were randomly divided into four groups (ten rats each):

Group I (Control group): The rats of this group received a single intraperitoneal injection of $1 \mathrm{ml} /$ $\mathrm{kg}$ body weight citrate buffer (0.1 M; pH 4.5) under ether anesthesia (Ann Geethan and Prince, 2008).

Group II (Diabetic untreated group): The rats were fasted for $14 \mathrm{~h}$ and diabetes was induced by a single intraperitoneal injection of $40 \mathrm{mg} / \mathrm{kg}$ body weight STZ (Sigma Chemical Co., St. Louis,
MO, USA) freshly dissolved in $1 \mathrm{ml} / \mathrm{kg}$ body weight citrate buffer $(0.1 \mathrm{M}$; $\mathrm{pH} 4.5)$ under ether anesthesia. After the injection, the animals were given free access to water and food. Blood samples were obtained via vein puncture of tail vein. Fasting glycemia was measured by the glucose oxidase method using a clinical glucometer. Plasma glucose level greater than $300 \mathrm{mg} / \mathrm{dl}$ confirmed the occurrence of diabetes that was determined three days after the drug injection (Anu Geethan and Prince, 2008).

Group III (Insulin treated group): After confirmation of diabetes, rats received subcutaneous injection of human insulin (rDNA), (Mixtard ${ }^{\circledR} 30$, Novo Nordisk, Denmark) with a dose ( $5 \mathrm{IU} / \mathrm{kg}$ body weight/day) for four weeks. The rats received the last insulin dose 24 hours before being sacrificed (Pinheiro et al., 2011).

Group IV (Cod liver oil treated group): After confirmation of diabetes, rats received pure cod liver oil (Arctic Cod Liver Oil ${ }^{\circledR}$, Nordic Naturals, Inc., USA) with a dose $(60 \mathrm{mg} / \mathrm{Kg}$ body weight/ day) by intra-gastric intubation plus subcutaneous injection of human insulin ( $5 \mathrm{IU} / \mathrm{kg}$ body weight/ day) for four weeks (Marjan et al., 2012).

\section{Animal sacrifice}

At the end of the experimental period which was four weeks for all groups, the animals were sacrificed by ketamine over dose (Shredah and ElSakhawy, 2014).

\section{Specimen preparation}

\section{Hematoxylin and Eosin (H\&E) staining}

The sublingual salivary glands were dissected out and fixed immediately in $10 \%$ buffered formalin solution. Then the specimens were washed by tap water, dehydrated in ascending grades of ethyl alcohol, cleared in xylene and embedded in paraffin wax. Sections of 4-5 $\mu \mathrm{m}$ in thickness were obtained 
and mounted on clean glass slides. Tissue sections were then deparaffinized in xylene and rehydrated by ethanol series ending with pure $\mathrm{H} 2 \mathrm{O}$ (Millipore Corporation, Temecula, CA, USA) before histological staining with H\&E solutions (Sigma, St. Louis, MO, USA), to verify histological details (Shredah and El-Sakhawy, 2014).

\section{Immunohistochemistry}

Immunolabeling for detection of caspase-3 was performed on paraffin sections of 4-5 $\mu \mathrm{m}$ in thickness mounted on positively charged microscope slides. The set of sections were incubated in hot oven for 2 hours at $56^{\circ} \mathrm{C}$, deparaffinized in xylene and rehydrated by ethanol series ending with pure $\mathrm{H} 2 \mathrm{O}$ (Millipore Corporation, Temecula, CA, USA). After 5 minutes washing in phosphate buffered saline (PBS), sections were incubated in $0.05 \mathrm{mg} / \mathrm{ml}$ proteinase $\mathrm{K}$ in $0.05 \mathrm{M}$ Tris- $\mathrm{HCl}, 0.01 \mathrm{M}$ Ethylene diamine tetra acetic acid (EDTA) and $0.01 \mathrm{M} \mathrm{NaCl}$, $\mathrm{pH} 7.8$ for 10 minutes at $37^{\circ} \mathrm{C}$. After two washes with PBS, unmasking of the antigens was carried out using antigen retrieval citrate buffer solution for 10 minutes in boiling water. Then the sections were placed in a humid chamber and the endogenous tissue peroxidase was blocked with $3 \%$ hydrogen peroxide for 5 minutes. Incubation with bovine serum albumin for 20 minutes was performed to reduce unwanted nonspecific reactions. Without washing, the sections were incubated with the primary antibody overnight at $4{ }^{\circ} \mathrm{C}$. The primary antibody used was anti-caspase-3 active form (Millipore Corporation, Temecula, CA, USA) with dilution 1:100. In the next day, after washing in PBS, the sections were incubated with secondary universal antibody (Vectastain Universal Elite ABC-peroxidase kit, Vector Laboratories) and then with the AvidinBiotin complex (ABC) (Vectastain Universal Elite $\mathrm{ABC}$ kit, Vector Laboratories, Burlingame, CA, USA) according to the manufacturer's protocol. The substrate 3,3'-diaminobenzidine (DAB) was applied for the same amount of time on all labeled sections until development of desired brown color.
Finally, the sections were counter-stained with Mayer's hematoxylin (Sigma, St. Louis, MO, USA) for 30 seconds to visualize tissue topography. The negative control was obtained by omitting the primary antibody from the protocol outlined above (Shredah and El-Sakhawy, 2014).

Histological and immunohistochemical examinations were performed using light microscope (Olympus ${ }^{\circledast}$ BX 60, Tokyo, Japan) at (400 x) magnification.

\section{Fluorescence staining}

A set of sections from each group was stained using thioflavin-T (Sigma, St. Louis, MO, USA) to detect amyloid fibrils aggregation. Sections were incubated in hot oven for 15 minutes at $60^{\circ} \mathrm{C}$, deparaffinized in xylene and rehydrated in descending grades of ethanol to water. After washing in PBS, the sections were incubated with thioflavin-T solution $(0.5 \%$ thioflavin- $\mathrm{T}$ in $0.1 \mathrm{~N} \mathrm{HCl})$ at room temperature away from light for 10 minutes. The sections thereafter, were rinsed sequentially in PBS and deionized water (for 5 minutes each) and then cover slipped. In the absence of amyloid fibrils, the dye fluoresced faintly at the excitation and emission maximum of 350 and $438 \mathrm{~nm}$, respectively. In the presence of amyloid fibrils, there was a bright fluorescence with this dye at the excitation and emission maximum of 450 and 482nm, respectively (Shen et al., 2011). All sections were examined under a dark field ultraviolet fluorescence microscope (Olympus ${ }^{\circledR}, \mathrm{BX} 41$, Tokyo, Japan) at (400 x) magnification.

\section{Histomorphometric analysis}

The data were obtained using Leica Qwin 500 image analyzer computer system (England). The image analyzer consisted of a coloured video camera, coloured monitor, hard disc of IBM personal computer connected to the microscope and controlled by Leica Qwin 500 software. The image analyzer was first calibrated automatically to convert the measurement units (pixels) produced by the image analyzer program into actual micrometer $(\mu \mathrm{m})$ units. 
In the immunohistochemistry treated sections and fluorescence stained sections, the image analysis system was used to assess the area percentage of caspase-3 immunoreactivity and thioflavin- $T$ positive staining respectively, in exceretory duct cells and surrounding connective tissue (C.T) stroma of the studied specimens. After grey calibration, the image was transformed into a grey delineated image to choose areas exhibiting positive reactivity/staining with accumulation of all grades of reactivity/staining (minimum, maximum and median grey). Areas of positive reaction/ staining were then masked by a blue binary color. Ten fields were measured for each specimen. The studied fields were measured under magnification 40x objective lens. Mean values were obtained for each specimen.

\section{Statistical analysis:}

Data obtained from histomorphometric analysis were analyzed using Statistical Program for Social Science (SPSS) version 20.0. Quantitative data were expressed as mean \pm standard deviation (SD). Qualitative data were expressed as frequency and percentage.

\section{The following tests were done:}

- A one-way analysis of variance (ANOVA) when comparing between more than two means.

- Tukey's Post Hoc test: Least Significant Difference (LSD) was used for multiple comparisons between different variables.

- The confidence interval was set to $95 \%$ and the margin of error accepted was set to $5 \%$. So, the p-value was considered significant as the following:

- Probability (P-value)

- P-value $\leq 0.05$ was considered significant.

- P-value $\leq 0.001$ was considered as highly significant.

- P-value >0.05 was considered insignificant.

\section{RESULTS}

\section{H \& E}

\section{Gp I Control group:}

The excretory ducts were observed interlobular, apparently large in size and lined by pseudostratified columnar epithelial cells. The ducts were surrounded by fibrous connective (C.T) stroma. Neighboring blood vessels (BVs) adjacent to the ducts were lined by thin endothelial cells. (Fig. 1 a).

\section{GP II diabetes}

The excretory duct cells showed loss of pseudostratification. Some lining cells nuclei were pyknotic, others were hyperchromatic or karyolytic. Cells with signs of degeneration were also observed. Stagnated secretion with desquamated cells have been noticed in the lumen of some ducts. The BVs adjacent to the excretory ducts were dilated, lined by swollen endothelial cells and engorged with RBCs. Few extravasated RBCs could be detected. Apparent decrease in the fibrous C.T surrounding the excretory ducts together with inflammatory cells infiltration could be observed. In addition, areas of hyalinization and large areas of degeneration in the C.T have been also noticed (Fig. 1 b).

\section{GP III Insulin}

Most of the excretory duct cells were pseudostratified columnar epithelial cells. However, some cells showed loss of pseudostratification. Stagnated secration could be noticed in some ducts. Few lining cells nuclei were pyknotic hyperchromatic and karyolytic. The BVs adjacent to the excretory ducts appeared somewhat dilated, lined by somewhat swollen endothelial cells and engorged with RBCs. Extravasated RBCs in the surrounding connective tissue were also observed. There was apparent improvement of the fibrous C.T surrounding the excretory ducts with few inflammatory cells. However areas of hyalinization and signs of degeneration were still detected. (Fig. 1 c). 


\section{Group IV ( Insulin +Cod liver oil treated group)}

The excretory ducts were observed interlobular, apparently large in size and lined by pseudostratified columnar epithelial cells except very few areas with loss of pseudostratification. In comparison to the previous groups, very few pyknotic and hyperchromatic nuclei were detected. The ducts lumina were almost empty and showed apparently varied diameters. The ducts were surrounded by fibrous C.T stroma with few inflammatory cells.
Moreover, apparently small areas of degeneration in the C.T could be observed. The neighboring BVs adjacent to the ducts had apparently small diameter and they were lined by thin endothelial cells (Fig. 1 d).

\section{Immunohistochemical results}

Nuclear and/or cytoplasmic caspase-3 expressions were detected in the ductal cells as well as the C.T cells of Groups I, II, III and IV with different patterns of reactivity.

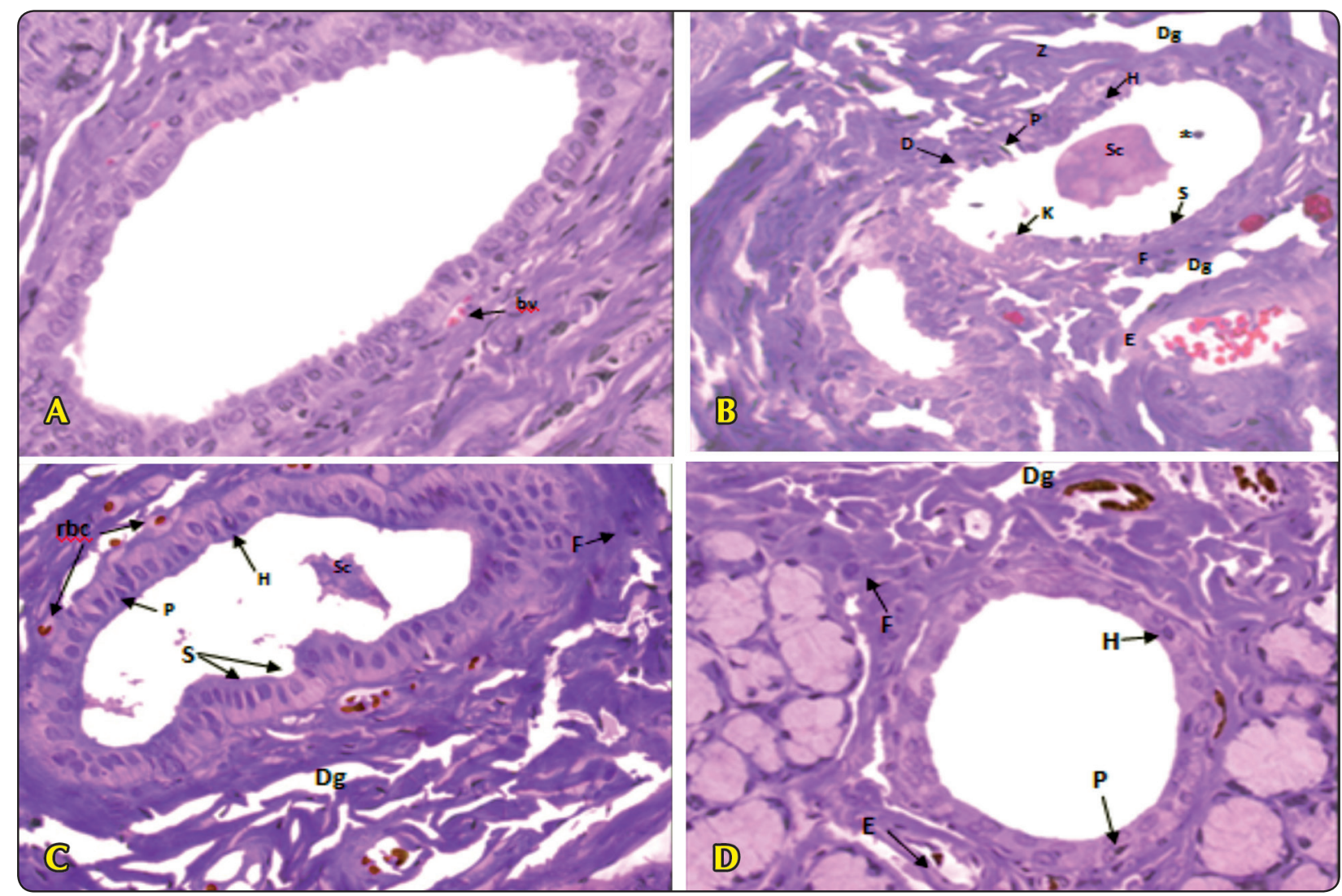

Fig. (1): Photomicrographs of H\&E sections: (a) Control group (I) showing: normal architecture of the excretory duct surrounded by fibrous C.T stroma. Normal BVs (bv), (the original magnification x400). (b)- Diabetic untreated group (II) showing: loss of pseudostratification $(\mathrm{S})$. Pyknotic $(\mathrm{P})$, hyperchromatic $(\mathrm{H})$ and few karyolytic nuclei. Cells with signs of degeneration (D). Desquamated cells (*). Homogenous stagnated secretion (Sc).. The dilated BVs are lined by swollen endothelial cells (E) and engorged with RBCs. Extravasated RBCs. Inflammatory cells infiltration (F), Areas of hyalinization (Z) and Apparently large areas of degeneration (Dg) in the C.T, (the original magnification x400). (c)- Insulin treated group (III) showing: Some cells devoid of pseudostratification $(\mathrm{S})$. Pyknotic $(\mathrm{P})$ and hyperchromatic $(\mathrm{H})$ nuclei. Stagnated secretion in the duct lumen $(\mathrm{Sc})$. Extravasated RBCs ( $\mathrm{rbc}$ ). Apparent increase of the fibrous C.T. except for Some inflammatory cells (F) and areas of degeneration (Dg). (the original magnification $\mathrm{x} 400)$. (d)- Cod liver oil-Insulin combined group (IV) showing: Almost normal architecture of the excretory duct . However, very few cells with pyknotic $(\mathrm{P})$ and hyperchromatic $(\mathrm{H})$ nuclei were observed. Very few inflammatory cells (F). Apparently small areas of degeneration (Dg) in the C.T. The BVs have apparently small diameter and lined by thin endothelial cells (E), (the original magnification $\mathrm{x} 400$ ). 
In Group I, the cells showed negative nuclear immunohistochemical expression for caspase-3 however, mild expression was observed in the cytoplasm of few cells (Figs. 2 a).

On contrary, Group II showed a strong immunoexpression for caspase-3 in the nuclei and cytoplasm of ductal cells and the C.T cells (Fig. 2b).

In Group III, moderate immuno-expression was detected in most of the nuclei and in the cytoplasm of the excretory duct cells and the C.T cells (Fig. 2c).

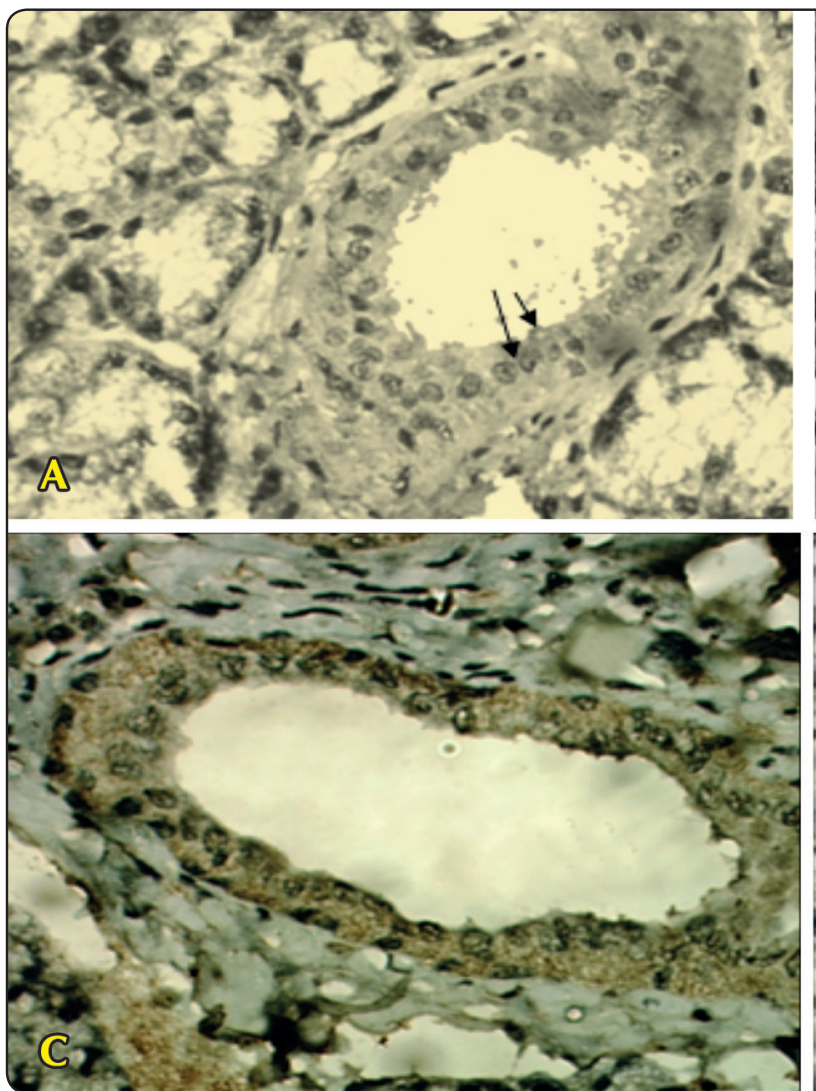

On the other hand, Group IV presented mild immuno-expression for caspase-3 in the cytoplasm of the ductal cells. In the C.T cells few cells with mild nuclear reaction were noticed. Negative nuclear immuno-expression was observed in the previous mentioned elements (Fig. 2d).

\section{Fluorescence staining results}

In Group I, the excretory duct and the C.T showed very faint fluorescence with thioflavin- $T$ stain (Figs. 3a).
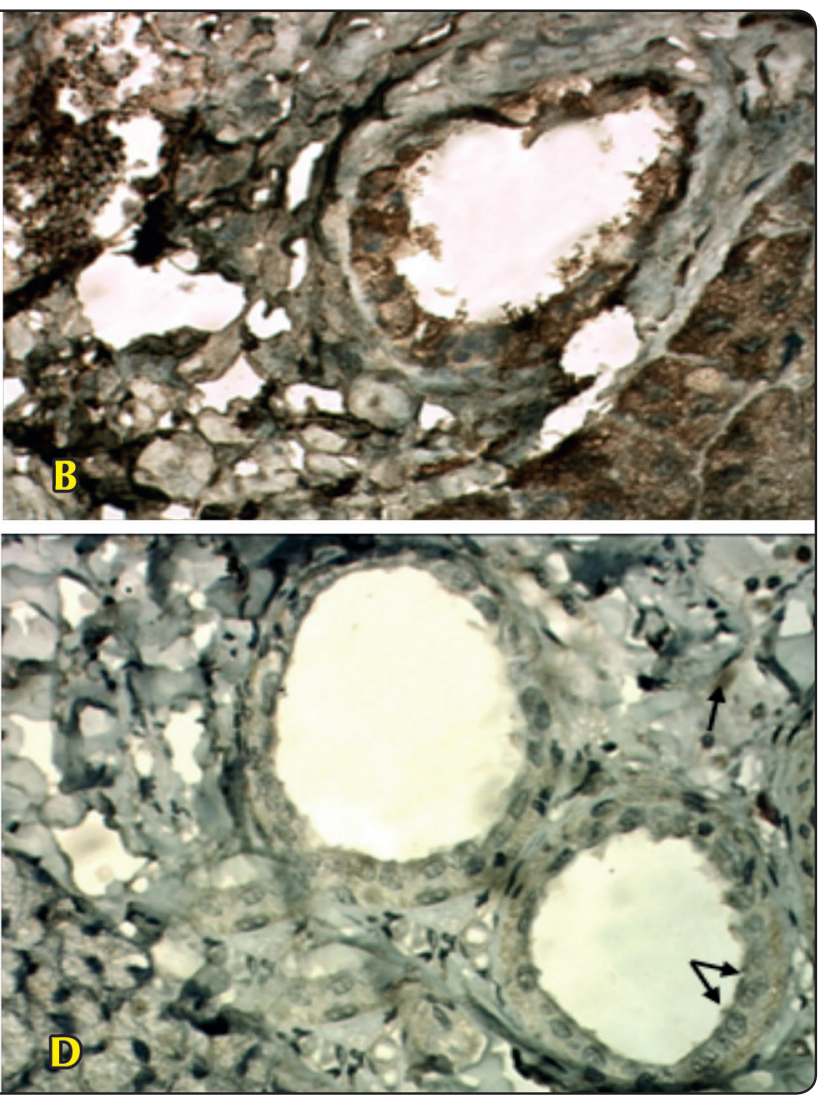

Fig. (2): Photomicrographs of caspase-3 immunolocalization: (a)- Control group (I) showing: almost negative nuclear \& cytoplasmic immunohistochemical expression for caspase-3. Mild expression in the cytoplasm of few cells in the excretory ducts (arrows). (the original magnification $\mathrm{x} 400$ ). (b)- Diabetic untreated group (II) showing: a strong immuno-expression for caspase-3 in the nuclei and cytoplasm of excretory duct cells and connective tissue cells.(the original magnification $\mathrm{x} 400$ ). (c)- Insulin treated group (III) showing: moderate immuno-expression in most of the nuclei and in the cytoplasm of the excretory duct cells as well as the connective tissue cells, (the original magnification x400). (d)- Cod liver oil-Insulin treated group (IV) showing: Almost negative nuclear immuno-expression for caspase-3 but mild immuno-expression in the cytoplasm of some excretory duct cells and the C.T cells (arrows).(the original magnification x400) 
On contrary, in Group II diffuse intracellular strong fluorescence with thioflavin-T was observed in the ductal cells. The C.T surrounding the excretory ducts showed diffuse strong fluorescence. Moreover, the basement membranes and the luminal surfaces of the excretory duct cells showed strong fluorescence. In addition, the stagnated salivary secretion in the ductal lumina showed strong fluorescence with thioflavin-T (Figs. 3b).

In Group III, diffuse intracellular moderate fluorescence with thioflavin-T was observed in some ductal cells. The C.T surrounding the excretory ducts showed moderate fluorescence except in scattered areas which showed strong fluorescence. (Fig. 3c).

On the other hand, in Group IV diffuse intracellular mild fluorescence with thioflavin-T was observed in some ductal cells as well as in the endothelium. Furthermore, the C.T surrounding the excretory ducts showed diffuse mild fluorescence. Moreover, the basement membranes and the luminal membranes of the excretory ducts showed mild fluorescence with thioflavin-T (Figs. 3d)

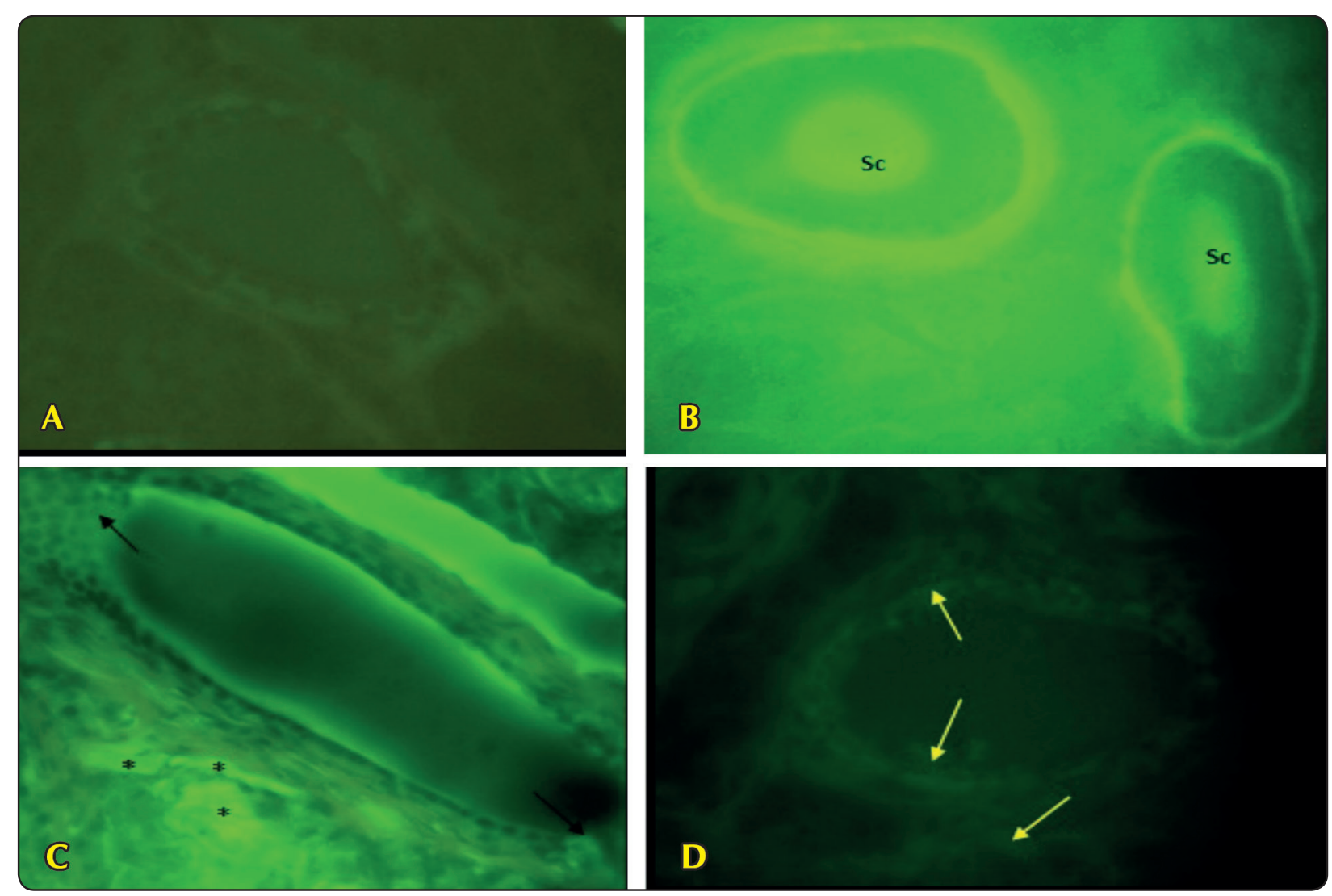

Fig. (3): Photomicrographs of thioflavin-T fluorescence staining: (a)- Control group (I) showing: the excretory duct and C.T have faint fluorescence with thioflavin-T stain, (the original magnification $x 400$ ). (b)- Diabetic untreated group (II) showing: diffuse \& strong cellular fluorescence with thioflavin-T in the excretory duct cells as well as the C.T stroma. The stagnated salivary secretion $(\mathrm{Sc})$ in the lumen of the excretory duct shows strong fluorescence with thioflavin- $\mathrm{T}$, (the original magnification x400). (c)- Insulin treated group (III) showing: Cellular moderate fluorescence with thioflavin-T in some excretory duct cells as well as the C.T.(arrows) Scattered areas with strong fluorescence (Astriks), (the original magnification x400). (d)Cod liver oil-Insulin treated group (IV) showing: diffuse intracellular mild fluorescence with thioflavin-T in some excretory duct cells and some areas of C.T (arrows). (the original magnification x400). 


\section{Statistical results:}

\section{a) Caspase-3 immunoreactivity}

The control group showed the least mean area percentage of caspase-3 immunoreactivity followed by CLO+ Insulin treated group, then insulin only treated group and the highest value was for the diabetic untreated group. One-way ANOVA showed highly significant difference between the studied groups. Tukey's post hoc test showed highly significant difference between each group when compared with the others (table 1 ).

\section{b) Thioflavin-T assay}

The control group showed the least mean area percentage of thioflavin-T fluorescence followed by CLO+ Insulin treated group, then insulin only treated group and the highest value was for the diabetic untreated group. One-way ANOVA showed highly significant difference between the studied groups. Tukey's post hoc test showed highly significant difference between each group when compared with the others (table 2).

TABLE (1) Comparison between groups according to Caspase 3 area expression.

\begin{tabular}{|c|c|c|c|c|c|c|}
\hline Caspase 3 area expression & Control & Diabetes & Insulin & CLO \& Insulin & $\mathbf{F}$ & p-value \\
\hline Mean \pm SD & $1.82 \pm 0.19$ & $67.85 \pm 2.07$ & $18.80 \pm 0.56$ & $3.52 \pm 0.47$ & \multirow{5}{*}{4485.773} & \multirow{5}{*}{$<0.001$} \\
\hline Range & $1.58-2.02$ & $65.8-71.04$ & $17.9-19.3$ & $2.8-4.1$ & & \\
\hline$\#$ & & $<0.001$ & $<0.001$ & 0.022 & & \\
\hline$\# \#$ & & & $<0.001$ & $<0.001$ & & \\
\hline \#\#\# & & & & $<0.001$ & & \\
\hline
\end{tabular}

F : ANOVA test; p-value $<0.05$ significant; -value $<0.001$ highly significant

\# Difference between control group and other group

\#\# Difference between diabetes group versus insulin and CLO \& insulin

\#\#\# Difference between insulin group versus CLO \& insulin

This table showed highly statistically significant difference between groups according to Caspase 3 area expression.

TABLE (2): Comparison between groups according to Thioflavin-T area expression.

\begin{tabular}{|c|c|c|c|c|c|c|}
\hline Thioflavin-T area expression & Control & Diabetes & Insulin & CLO \& Insulin & $\mathbf{F}$ & p-value \\
\hline Mean \pm SD & $0.10 \pm 0.03$ & $41.82 \pm 2.47$ & $20.18 \pm 1.42$ & $1.86 \pm 0.23$ & \multirow{5}{*}{927.465} & \multirow{5}{*}{$<0.001$} \\
\hline Range & $0.07-0.14$ & $38.8-44.2$ & $18.8-22.16$ & $1.5-2.1$ & & \\
\hline \# & & $<0.001$ & $<0.001$ & 0.069 & & \\
\hline \#\# & & & $<0.001$ & $<0.001$ & & \\
\hline \#\#\# & & & & $<0.001$ & & \\
\hline
\end{tabular}

F : ANOVA test; p-value $<0.05$ significant; p-value $<0.001$ highly significant

\# Difference between control group and other group

\#\# Difference between diabetes group versus insulin and CLO \& insulin

\#\#\# Difference between insulin group versus CLO \& insulin

This table shows highly statistically significant difference between groups according to Thioflavin-T area expression. 


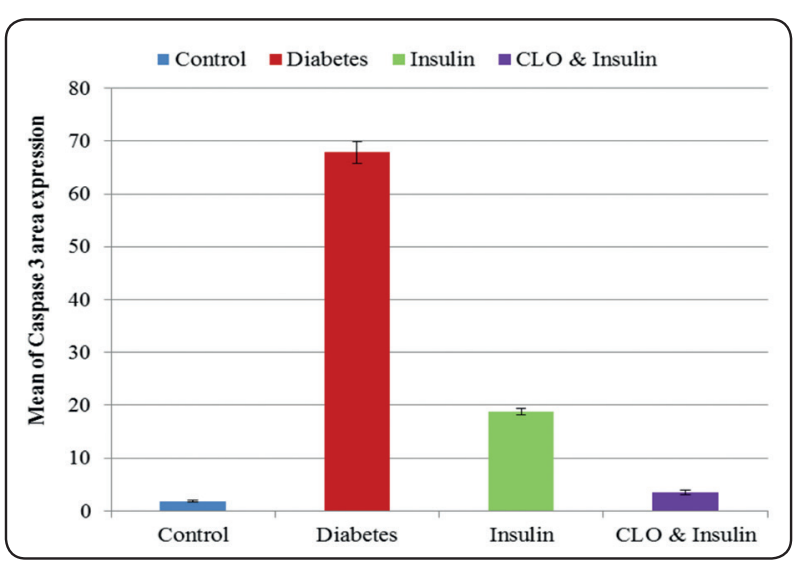

Fig. (4) Bar chart omparison between groups according to Caspase 3 area expression.

\section{DISCUSSION}

Generally the salivary glands are to some extent similar in structure and function to the exocrine pancreas. Also it could be more liable to oxidative damage via reactive oxygen species (ROS) through presenting a predominantly aerobic metabolism (Humphrey and Wiliamson, 2001).

A rat model is used here for type II diabetes. This may show similarity to clinical pathogenesis seen in humans who have insulin resistance and partial pancreatic $\beta$-cell dysfunction. In the present study this was achieved by injection of $40 \mathrm{mg} / \mathrm{kg}$ body weight STZ which is the ideal dose for development of animal model with type II diabetes (Islam and Loots, 2009). Furthermore, Rodent salivary glands used in animal experiments show an almost similar histology to the human glands (Osamu Amano, et al., 2012).

Excretory duct is a major interlobar duct of large diameter and surrounded by the vascular connective tissue stromal septa of the gland. Thus, it could be a good histological representative study model to the duct system.

The current study revealed various pathological changes in the glandular excretory duct architecture, C.T stroma and BVs in the diabetic untreated group

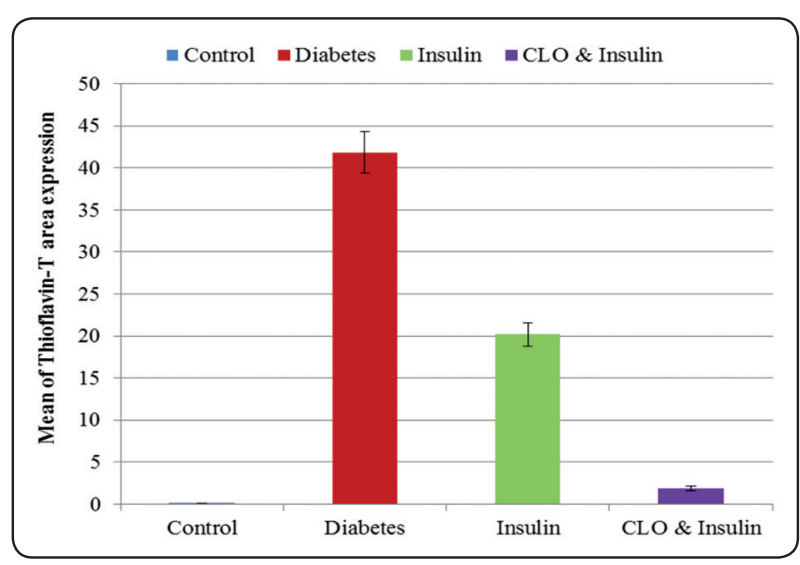

Fig. (5) Bar chart between groups according to Thioflavin-T area expression

(Group II) when compared to the control group. These changes include nuclear changes in form of; hyperchromatism, pyknosis and karyolysis. Moreover, some areas of complete degeneration of the ductal cells were reported. Desquamated cells in the ductal lumina and dilated BVs with swollen endothelial cells were also observed. These findings are considered as pre-necrotic changes that are relevant to the persistent hyperglycemia (Parlak et al., 2014; Shredah and El-Sakhawy, 2014).

The poorly defined ductal cells outlines detected in Group II of this study could be attributed to the fact that lipids are the most sensitive to free radicals. Thus the free radicals produced due to persistent hyperglycemia could result in changes in the acyl fatty acids composition which are the main components of the phospholipid bilayer. Thus, Changes in the phospholipid fatty acid composition of ductal cell membranes will result in changes in the collective physicochemical properties, such as flexibility and integrity of the lipid bilayer (Mahay et al., 2004; Leite et al., 2014).

The stagnated salivary secretion in the lumina of the ducts of Group II, could be explained according to the fact that diabetes not only affects the structural elements of the gland but also affects its function (Sabino-Silva et al., 2013). 
The histological results of Group II in this study revealed dilated BVs; lined by swollen endothelial cells and engorged with RBCs. Furthermore, few extravasated RBCs as well as inflammatory cells infiltration in the C.T surrounding the excretory ducts were detected. These observations could be attributed to the fact that the dilatation and congestion of the BVs might be a part of inflammatory response to bring more blood to the areas of degeneration (Moubarak, 2008). Moreover, during hyperglycemia, increased ROS and diacylglycerol (DAG) stimulate protein kinase $\mathrm{C}$ (PKC) in the vascular tissue. Both PKC and ROS cause loss of permeability that leads to swelling of the endothelium (Evcimen and King, 2007).

In the current study, Group II showed apparent decrease in the fibrous C.T surrounding the excretory ducts, in addition to hyalinization and degeneration that were noticed in some areas. Moreover, fibroblasts showing signs of degeneration were noticed. Our findings could be explained according to the fact that advanced glycation end-products (AGEs) induce fibroblast (the matrix producing cell) apoptosis, which is mediated through caspase-3 and signaled through both caspase- 8 and caspase- 9 activities leading to suppression of collagen synthesis and excessive collagenolytic activity. Not only the fibroblasts, but also the inflammatory cells undergo apoptosis which is mediated through caspase-3 (Riedl and Shi, 2004; Alikhani et al., $2005 a)$.

Our imunohistochemical results confirm our histological findings observed in Group II of the current study, in which caspase-3 antibodies revealed intense positive reaction to activated caspase- 3 in the nuclei and cytoplasm of the ductal cells, C.T cells as well as the endothelial cells. Our findings could be attributed to the fact that the toxicity of STZ is related to the inhibition of the enzyme O-GlcNAcase (N-acetyl-D-glucosaminidase) which removes protein linked GlcNAc (Liu et al., 2000; Konrad et al., 2001; Szkudelski, 2001). The increase in intracellular levels of proteins modified by GlcNAc results in cell death by apoptosis (Konrad et al., 2001). Furthermore, the metabolic disturbances associated with diabetes can lead to activation of the polyol pathway, high levels of the cytokine tumor necrosis factor (TNF)- $\alpha$, the formation of AGEs, high levels of PKC as well as activation and enhanced oxidative stress (Asnaghi et al., 2003). The activation of these pathways may be especially important in initiating events linked to inflammation and apoptosis (Dagher et al., 2004; Xu et al., 2004).

Another explanation for the mechanism is through the increased oxidative stress, which is one of the common pathogenic factors of diabetes complications. It leads to the formation of excess ROS which lead to severe oxidative damage of the cell's components like lipids, proteins and DNA by inhibiting many of the enzymes involved in DNA synthesis. These pathways lead to one result which is apoptosis through activation of caspases (Riedl and Shi, 2004).

In the present study, the mild immuno-expression for caspase-3 observed in the control group (Group I) might be attributed to the physiological cell death (Shredah and El-Sakhawy, 2014).

Thioflavin-T was used in the current study to detect amyloid fibrils. The results showed strong fluorescence to thioflavin-T in group II. Amyloidosis is the case in which protein aggregation produces ordered polymers that form protofilaments and then fibrils about $10 \mathrm{~nm}$ in diameter. (Millucci et al., 2014). Thioflavin- $T$ is a benzothiazole dye that exhibits enhanced fluorescence upon binding to amyloid fibrils and is commonly used to diagnose amyloid fibrils, both ex vivo and in vitro (Khurana et al., 2005).

It was reported by Hidayat et al., (2014) that Diabetes increases the degree of both lipid peroxidation and protein glycosylation, also it elevates the levels of total serum cholesterol (TC), triglycerides and low-density lipoprotein (LDL). Furthermore, serum biomarkers related to type II diabetes seem to correlate with inflammatory 
responses and lipid metabolism pathways. Two of these important apolipoprotein associated serum biomarkers are serum amyloid A (SAA) and serotransferrin (TRFE). It was reported that increased expression of SAA in serum of diabetic patients was also related to SAA related inflammatory mediator, $\mathrm{C}$-reactive protein (CRP). It was suggested that diabetes-related serum biomarkers such as SAA and TRFE, are present in saliva. These biomarkers may be released from the serum through a salivary gland apparatus (Li et al., 2008).

Amyloids interact with constituents of basement membranes such as perlecan, laminin and agrin. Moreover, cytoplasmic membrane and organelles as (endoplasmic reticulum, mitochondria and nuclear membrane) were also interacted with. Vascular abnormalities including endothelial enlargement, basement membrane modifications and vascular proliferation were noticed with amyloidosis (García-García et al., 2002).

Although type II diabetes is non-insulindependent DM however, it was reported that over time most patients with type II diabetes experience progressive $\beta$-cell dysfunction and will require insulin therapy for satisfactory glycemic control (American Diabetes Association, 2009). In the current study, the insulin dose applied was based on the proposal that the best glycemic control was obtained with the dose $5 \mathrm{IU} / \mathrm{kg}$ body weight/day (Pinheiro et al., 2011).

In the current study the histological, immunohistochemical, fluorescence and statistical results of Group III were better than those of Group II. Our results came in accordance with those of Take et al. (2007) who reported better structural and functional integrity of parotid gland after insulin treatment. The authors attributed their results to the fact that insulin stimulates several processes such as carbohydrate transport, protein synthesis and cholecystokinin (CCK) receptor synthesis; CCK has a role in increasing the activity of $\alpha$-amylase in parotid gland. Moreover, Take et al. (2007) reported that insulin treatment for one week showed better fatty acids profile and lipid droplet contents of the acinar cells. The researchers attributed their results to the regulation of lipid metabolism by insulin.

However our observations revealed that insulin did not completely inhibit the complications of diabetes. This finding was in agreement with those of Zobali et al. (2002) as well as Jain et al. (2006) who stated that "insulin did not completely inhibit the abnormalities in the oxidative metabolism of the parotid glands in diabetic rats that still suffered an oxidative insult". The authors attributed their results to the fact that insulin treatment partially inhibits lipid peroxidation, and also it is not strong enough to prevent excessive protein glycation, which can increase tissue oxidative stress.

The histological, immunohistochemical, fluorescence and statistical results of Group IV in the current study showed better criteria and histological findings. This means that when CLO combined with insulin, the abnormalities in the oxidative metabolism recorded in Group II were markedly diminished if compared to the effect of insulin alone in Group III. Our results could be referred to the fact that $\omega-3$ fatty acids in CLO rather than other sources of $\omega-3$ fatty acids, not only significantly attenuate the chemically-induced DM sequelae, but also act on suppressing the production of cytokines and inhibit the production of the proinflammatory leukotrienes B2 and C4 by activated leukocytes. Furthermore, $\omega-3$ fatty acids considerably inhibit the elevation of TNF- $\alpha$, IL1, IL-6 and CRP (Hamdy et al., 2007; Tsitouras et al., 2008). Moreover, $\omega-3$ fatty acids maintain the antioxidant status in a normal range through significant increase in the activities of antioxidant enzymes such as superoxide dismutase (SOD) by up regulating gene expression of antioxidant enzymes and down regulating gene associated with production of ROS (Soltan, 2012; Hussein et al., 2014). Consequently, $\omega-3$ fatty acids in turn can suppress the formation of oxygen free radicals, lipid peroxides as well as aldehydes (Hamdy et al., 2007; 
Hussein et al., 2014). In addition, it was recorded that, $\omega-3$ fatty acids showed an expressive increase of $\alpha$-amylase activity in parotid gland through stimulation of adenylate-cyclase activity (Leite et al., 2014).

This may be attributed to that $\omega-3$ fatty acids inhibit the increase of TC as well as LDL levels and supress triglyceridemia through enhanced triacylglycerol lipolysis (Hamdy et al., 2007). Also, it was documented that $\omega-3$ fatty acids improve insulin action in liver by reducing intracellular fat long chains content. Such mechanism is not only responsible for enhanced fatty acid utilization but also contributes to improvement in insulin sensitivity (Tsitouras et al., 2008). $\omega-3$ fatty acids may also play an important role in up regulation of insulin synthesis (by ß-cells) and its subsequent transport extracellularly through inhibiting oxidative stress formation. Consequently, $\omega-3$ fatty acids have inhibitory effect on increased plasma glucose and improve glucose utilization (Soltan, 2012).

Further investigations are required to prove; whether or not CLO intake can prevent type II diabetes development. Also, to examine the effect of omega-3 supplements administration as an alternative therapy to insulin resistant patients. Moreover, the availability of co-administration of omega-3 supplementations with chemotherapeutic agents and other conditions that are associated with elevated levels of ROS.

\section{CONCLUSIONS}

This study had demonstrated that diabetes has deleterious effect on the structure and function of excretory duct of sublingual salivary glands. Moreover, it plays a major role in tissue damage through development of amyloidosis.

Insulin as a sole treatment couldn't completely inhibit the complications of diabetes. However, when combined with CLO showed a great potentiality to minimize the abnormalities caused by diabetes.

\section{REFERENCES}

1. Alikhani, Z., Alikhani, M., Boyd, C., Nagao, K., Trackman, P. and Graves, D.: Advanced glycation end products enhance expression of pro-apoptotic genes and stimulate fibroblast apoptosis through cytoplasmic and mitochondrial pathways. J. Biol. Chem.; 280: 12087-12095, 2005 (a).

2. American Diabetes Association: Diagnosis and classification of diabetes mellitus. Diab. Care; 32: S62-S67, 2009.

3. Ann Geethan, P.K.M. and Prince, P.S.M.: Antihyperlipidemic effect of D-Pinitol on streptozotocin-induced diabetic wistar Rats. J. Biochem. Mol. Toxic.; 22 (4): 220-224, 2008 .

4. Asnaghi, V., Gerhardinger, C., Hoehn, T., Adeboje, A. and Lorenzi, M.: A role for the polyol pathway in the early neuroretinal apoptosis and glial changes induced by diabetes in the rat. Diabetes; 52: 506-511, 2003.

5. Aydin, S.: A comparison of ghrelin, glucose, alpha-amylase and protein levels in saliva from diabetics. Biochem. Mol. Biol.; 40: 29-35, 2007.

6. Border, M.B., Schwartz, S., Carlson, J., Dibble, C.F., Kohltfarber, H., Offenbacher, S., Buse, J.B. and Bencharit, S.: Exploring salivary proteomes in edentulous patients with type 2 diabetes. Mol. Biosys.; 8: 1304-1310, 2012.

7. Burrows, N.R., Geiss, L.S., Engelgaum, M.M. and Acton, K.J.: Prevalence of diabetes among Native Americans and Alaska Natives, 1990-1997: an increasing burden. Diab. Care; 23 (12): 1770-1786, 2000.

8. Carda, C., Mosquera-Lloreda, N., Salom, L., Gomez de Ferraris, M.E. and Peydró, A.: A structural and functional salivary disorders in type 2 diabetic patients. Oral Med. Pathol.; 11: 309-314, 2006.

9. Chapple, I.L.C. and Genco, R.: Diabetes and periodontal diseases: consensus report of the joint EFP/AAP workshop on periodontitis and systemic diseases. J. Periodontol.; 84 (4): S106-S112, 2013.

10. Dagher, Z., Park, Y.S., Asnaghi, V., Hoehn, T., Gerhardinger, C. and Lorenzi, M.: Studies of rat and human retinas predict a role for the polyol pathway in human diabetic retinopathy. Diabetes; 53: 2404-2411, 2004.

11. Evcimen, N.D. and King, G.L.: The role of protein kinase $\mathrm{C}$ activation and the vascular complications of diabetes. Pharmacol. Res.; 55 (6): 498-510, 2007.

12. Fabian, T.K., Fejerdy, P. and Csermely, P.: Salivary genomics, transcriptomics and proteomics: the emerging concept 
of the oral ecosystem and their use in the early diagnosis of cancer and other diseases. Curr. Genomics; 9: 11-21, 2008.

13. García-García, M., Mourad, G., Durfort, M., GarcíaValero, J. and Argilés, À.: Vascular involvement and cell damage in experimental AA and clinical $\beta_{2}$-microglobulin amyloidosis. Nephrol. Dial. Transplant.; 17 (8): 1450$1456,2002$.

14. Gregersen, N. and Bross, P.: Protein misfolding and cellular stress: an overview. Methods Mol. Biol.; 648: 3-23, 2010 .

15. Hamdy, M.S., Moselhy, S.S., Makhlouf, A.A. and Fathy, A.H.: Cod liver oil in chemically-induced diabetes mellitus in rats. J. Biol. Res.; 7: 59-65, 2007.

16. Hidayat, M., Tahir, M. and Shoro, A.A.: Melatonin preserves the morphology of parotid gland damaged by streptozotocin-induced diabetes. J. Postgrad. Med. Inst.; 28 (2): 128-132, 2014.

17. Hirtz, C., Chevalier, F., Sommerer, N., Raingeard, I., Bringer, J., Rossignol, M. and de Périere Deville, D.: Salivary protein profiling in type I diabetes using twodimensional electrophoresis and mass spectrometry. Clin. Proteomics; 2: 117-127, 2006.

18. Humphrey, S.P. and Williamson, R.T.: A review of saliva: normal composition, flow and function. J. Prosth. Dent.; 85 (2): 162-169, 2001.

19. Hussein, J.S., El-Khayat, Z., Morsy, S., Oraby, F. and Singer, G.: The effect of fish oil on oxidant/antioxidant status in diabetic rats through the reduction of arachidonic acid in the cell membrane. Int. J. Pharm. \& Pharm. Sci.; 6 (2): 196-199, 2014.

20. Iannuzzi, C., Irace, G. and Sirangelo, I.: The effect of glycosaminoglycans (GAGs) on amyloid aggregation and toxicity. Molecules; 20: 2510-2528, 2015.

21. Islam, M.S. and Loots, D.T.: Experimental rodent models of type 2 diabetes: a review. Methods Find. Exp. Clin. Pharmacol.; 31: 249-261, 2009.

22. Jain, S., Pandhi, P., Singh, A.P. and Malhotra, S.: Efficacy of standardized herbal extracts in type 1 diabetes - an experimental study. Afr. J. Trad. CAM; 3 (4): 23-33, 2006.

23. Jorgensen, M.F., Bjergaard, P. and Barchjohnsen, K.: Diabetes and impaired glucose tolerance among the Inuit population of Greenland. Diab. Care; 25 (10): 1766-1771, 2002
24. Khurana R., Coleman C., Ionescu-Za Carter S.A., Krishna V., Grover R.K. et al.: Mechanism of thioflavin-T binding to amyloid fibrils "J. Struct. Biol.; 151 (3): 229-238, 2005.

25. Kidambi, S. and Patel, S.B.: Diabetes mellitus: considerations for dentistry. J. Am. Dent. Assoc.; 139: 8-18, 2008.

26. Konrad, R.J., Mikolaenko, I., Tolar, J.F., Liu, K. and Kudlow, J.E.: The potential mechanism of the diabetogenic action of streptozotocin: inhibition of pancreatic B-cell O-GlcNAc-selective N-acetyl- $\beta-D-$ glucosaminidase. Biochem. J.; 356: 31-41, 2001.

27. Leite, M.F., Lima, A.M., Sun Kang, S.J., Santos, M.T. and Otton, R.: Effect of astaxanthin and fish oil on enzymatic antioxidant system and $\alpha$-amylase activity of salivary glands from rats. Braz. J. Oral Sci.; 13 (1): 58-63, 2014.

28. Liu, K., Paterson, A.J., Chin, E. and Kudlow, J.E.: Glucose stimulates protein modification by O-linked GlcNAc in pancreatic $B$ cells: linkage of O-linked GlcNAc to $B$ cell death. Proc. Natl. Acad. Sci. USA; 97: 2820-2825, 2000.

29. Li, R.X., Chen, H.B., Tu, K., Zhao, S.L., Zhou, H., Li, S.J., Dai, J., Li, Q.R., Nie, S., Li, Y.X., Jia, W.P., Zeng, R. and Wu, J.R.: Localized-statistical quantification of human serum proteome associated with type 2 diabetes. PLOS One; 3 (9): 1-13, 2008.

30. Lordan, S., Ross, R.P. and Stanton, C.: Marine bioactives as functional food ingredients: potential to reduce the incidence of chronic diseases. Mar. Drugs; 9: 1056-1100, 2011.

31. Mahay, S., Adeghate, E., Lindley, M.Z., Rolph, C.E. and Singh, J.: Streptozotocin-induced type 1 diabetes mellitus alters the morphology, secretory function and acyl lipid contents in the isolated rat parotid salivary gland. Mol. Cell. Biochem.; 261: 175-181, 2004.

32. Marjan, A., Shariar, E., Rouhollah, H., Jalaledin, M., Habibolah, P., et al.: Effect of short and long-term treatment with omega-3 fatty acids on scopolamine-induced amnesia. Iranian J. Pharm. Res.; 11: 533-540, 2012.

33. Mckim, S.E., Kono, A., Gabele, E., Uesugi, T., Froh, M., Sies, H., Thurman, R.G. and Arteel, G.E.: Cocoa extract protects against early alcohol induced liver injury in the rat. Arch. Biochem. Bioassay; 406: 40-46, 2002.

34. Merlini, G. and Bellotti, V.: Molecular mechanisms of amyloidosis. N. Engl. J. Med.; 349: 583-596, 2003.

35. Millucci, L., Ghezzi, L., Bernardini, G., Braconi, D., Lupetti, P., Perfetto, F., Orlandini, M. and Santucci, A.: Diagnosis of secondary amyloidosis in alkaptonuria. Diagn. Pathol.; 9: 1-9, 2014. 
36. Moubarak, R.: The effect of hypercholesterolemia on the rat parotid salivary glands (histopathological and immunohistochemical study). CDJ; 24 (I): 19-28, 2008.

37. Negrato, C.A. and Tarzia, O.: Buccal alterations in diabetes mellitus. Diabetol. Metab. Syndr.; 15: 1-11, 2010.

38. Obici, L., Perfetti, V., Palladini, G., Moratti, R. and Merlini, G.: Clinical aspects of systemic amyloid diseases. Biochim. Biophys. Acta; 1753: 11-22, 2005.

39. Osamu Amano, Kenichi Mizobe, Yasuhiko Bando and Koji Sakiyama: Anatomy and Histology of Rodent and Human Major Salivary Glands. Acta Histochem Cytochem:. 31; 45(5): 241-250, 2012.

40. Parlak, S.N., Tatar, A., Keles, O.N., Selli, J., Can, I. and Unal, B.: Effects of menopause and diabetes on the rat parotid glands: a histopathological and stereological study. Int. J. Med. Sci. Pub. H.; 3 (6): 749-755, 2014.

41. Pinheiro, L.S., Melo, A.D., Andreazzi, A.E., Júnior, L.C.C., Costa, M.B. and Garcia, R.M.G.: Protocol of insulin therapy for streptozotocin-diabetic rats based on a study of food ingestion and glycemic variation. Scand. J. Lab. Anim. Sci.; 38 (2): 117-127, 2011.

42. Rao, P.V., Reddy, A.P., Lu, X., Dasari, S., Krishnaprasad, A., Biggs, E., Roberts, C.T. and Nagalla, S.R.: Proteomic identification of salivary biomarkers of type-2 diabetes. J. Proteome Res.; 8: 239-245, 2009.

43. Riedl, S.J. and Shi, Y.: Molecular mechanisms of caspase regulation during apoptosis. Nat. Rev. Mol. Cell Biol.; 5: 897-907, 2004

44. Sabino-Silva, R., Okamoto, M.M., David-Silva, A., Mori, R.C., Freitas, H.S. and Machado, U.F.: Increased SGLT1 expression in salivary gland ductal cells correlates with hyposalivation in diabetic and hypertensive rats. Diabetol. Metab. Syndr.; 5: 64-68, 2013.

45. Shen, D., Coleman, J., Chan, E., Nicholson, T.P., Dai, L., Sheppard, P.W. and Patton, W.F.: Novel cell- and tissuebased assays for detecting misfolded and aggregated protein accumulation within aggresomes and inclusion bodies. Cell Biochem. Biophys.; 60: 173-185, 2011.

46. Shredah, M. and El-Sakhawy, M.A.: Immunohistochemical expression of activated caspase- 3 in the parotid salivary glands of rats after long administration of Myristica fragrans. Int. J. Adv. Res.; 2 (12): 493-499, 2014.
47. Simonsen, T., Vartun, F.A., Lyngmo, V. and Nordoy, A.: A coronary heart disease, serum lipid, platelets and dietary fish in two communities in Northern Norway. Acta Medica Scand.; 222 (3): 237-243, 1987.

48. Skinner, M., Sanchorawala, V., Seldin, D.C., Dember, L.M., Falk, R.H., Berk, J.L., Anderson, J.J., O’Hara, C., Finn, K.T., Libbey, C.A., Wiesman, J., Quillen, K., Swan, N. and Wright, D.G.: High-dose melphalan and autologous stem-cell transplantation in patients with $\mathrm{AL}$ amyloidosis: an 8-year study. Ann. Intern. Med.; 140: 85-93, 2004.

49. Soltan, S.A.: The effects of varieties sources of omega-3 fatty acids on diabetes in rats. Food Nutr. Sci.; 3: 1404 1412, 2012.

50. Szkudelski, T.: The mechanism of alloxan and streptozotocin action in $\beta$ cells of the rat pancreas. Physiol. Res.; 50: 536-546, 2001.

51. Take, G., Ilgaz, C., Erdogan, D., Ozogul, C. and Elmas, C.: A comparative study of the ultrastructure of submandibular, parotid and exocrine pancreas in diabetes and fasting. SMJ; 28 (1): 28-35, 2007.

52. Tsitouras, P.D., Gucciardo, F., Salbe, A.D., Heward, C. and Harman, S.M.: High omega-3 fat intake improves insulin sensitivity and reduces CRP and IL6, but does not affect other endocrine axes in healthy older adults. Horm. Metab. Res.; 40: 199-205, 2008.

53. Westermark, P., Benson, M.D., Buxbaum, J.N., Cohen, A.S., Frangione, B., Ikeda, S., Masters, C.L., Merlini, G., Saraiva, M.J. and Sipe, J.D.: Amyloid: toward terminology clarification. Report from the Nomenclature Committee of the International Society of Amyloidosis. Amyloid; 12: $1-4,2005$.

54. Wong, D.T.: Salivary diagnostics powered by nanotechnologies, proteomics and genomics. J. Am. Dent. Assoc.; 137: 313-321, 2006

55. Xu, X., Zhu, Q., Xia, X., Zhang, S., Gu, Q. and Luo, D.: Blood-retinal barrier breakdown induced by activation of protein kinase $\mathrm{C}$ via vascular endothelial growth factor in streptozotocin-induced diabetic rats. Curr. Eye Res.; 28: 251-256, 2004.

56. Zobali, F., Avci, A., Canbolat, O. and Karasu, C.: Effects of vitamin A and insulin on the antioxidative state of diabetic rat heart: a comparison study with combination treatment. Cell Biochem. Funct.; 20 (2): 75-80, 2002. 